\title{
Contact mechanical problem for arbitrary 3D loading histories
}

\author{
V. V. Aleshin \& O. Bou Matar \\ Institute of Electronics, Microelectronics and Nanotechnologies, \\ Lille, France
}

\begin{abstract}
This communication is concerned with the method of memory diagrams developed for solving a problem of frictional elastic contact. Our goal is to establish a link between contact force and displacement for a general plane contact between two arbitrarily-shaped bodies; rolling and torsion are not considered. Description of mechanical interactions between two solids in contact in the presence of friction is a non-trivial task since the desired forcedisplacement relationships have hysteretic (memory-dependent) character. Arbitrarily changing applied force (or displacement) creates a cumbersome shear stress distribution in the contact zone that has to be adequately parameterized and accounted for. In that regard, it is suggested to consider, instead of complex shear stress distributions, a simpler functional form called memory diagram that contains the same memory information. We have established two integral relationships that link the force and displacement vectors with that internal functional dependency. The integral relationships are supplemented with two other evolution rules for memory diagrams that eventually follow from the Coulomb friction law. The memory diagram is updated with the help of these rules following a given force history. Then the calculated memory diagram is used to update the history of displacement i.e. to produce the desired forcedisplacement relationship.

Keywords: friction, contact mechanics, Hertz-Mindlin, frictional contact, frictional hysteresis, general loading history, arbitrary loading history.
\end{abstract}




\section{Introduction}

The history of the contact problem started in the 1880s when H. Hertz published the classical solution for two elastic spheres compressed by a normal force [1]. In absence of adhesion and plasticity, this solution is fully reversible. However, the addition of a tangential force and friction $[2,3]$ makes the problem hysteretic and memory-dependent. It was noted [3] that even a small tangential force acting on two pre-compressed spheres results in appearance of a slip annulus at the periphery of the contact circle where the surfaces are compressed weakly. The coexistence of the stick (central) and slip (peripheral) zones actually means mixed-type boundary conditions that correspond to zero local tangential displacement in the central region and, in the slip annulus, to the Coulomb friction law written for local tangential $(\tau)$ and normal $(\sigma)$ stresses, $\tau=\mu \sigma$ (here $\mu$ is friction coefficient considered as a constant for two contacting materials). The increase in the tangential force results in the slip propagation towards the contact centre. If now the tangential force starts decreasing, a new slip annulus develops at the contact periphery in which $\tau=-\mu \sigma$. Hence, the same values of the normal $(N)$ and tangential $(T)$ forces can correspond to different distributions of stresses and displacements in the contact zone. This fact explains a complex hysteretic character of the solution.

Allowing the normal force to evolve [4] adds a new complexity factor to the problem. The matter is that the slip zone always arises at the contact border where $\sigma=0$ and propagates inward, but, if the normal force increases, the contact border itself propagates outward. The result depends on the value of the derivative $d N / d T$.

Finally, the introduction of a general contact geometry instead of spherical profiles introduces even more complexity into the description. Indeed, for two rough surfaces, the contact zone consists of a multitude of contact spots having random geometry. For changing normal force, those contact spots can merge or split. Further, each of them supports slip and stick zones, and traction distribution in the stick zones can contain residual stresses left from the previous moments of evolution. With continuously varying normal and tangential loading, this complicated picture continuously evolves.

Here we propose a solution to such a general mechanical problem which is based on known works [4-9] as well as on original developments [10-14]. The geometric aspect of the problem can be successfully dealt with by using the Reduced Elastic Friction Principle (REFP, [5-9]) that, under some restrictions, makes it possible to replace an arbitrary contact geometry by a pair of axisymmetric profiles. Then, following the original Method of Memory Diagrams (MMD) we introduce an internal functional dependency called memory diagram that replaces a cumbersome traction distribution in the contact zone but contains the same information. Further, the MMD enables us to link the tangential force and tangential displacement via two integral equations each depending on the memory diagram. Finally, generalizing an incremental procedure established in [4], we develop an algorithm that produces a forcedisplacement relationship for an arbitrary loading history. 


\section{Reduced elastic friction principle}

The REFP [5-9] is an important theorem of contact mechanics which states that, for constant loading and for a wide range of contact geometries, the tangential force and displacement can be expressed through the normal force and displacement. This principle is illustrated in fig. 1 (on the left) for axisymmetric bodies. Consider two situations: first one when the system is loaded only by normal force $Q$, and second one when both force components, $N$ and $T$, are applied $(N>Q)$. The force values are chosen in such a way that the stick zone in the second case coincides with the contact zone in the first case. Then the tangential force and displacement in the second situation are given by
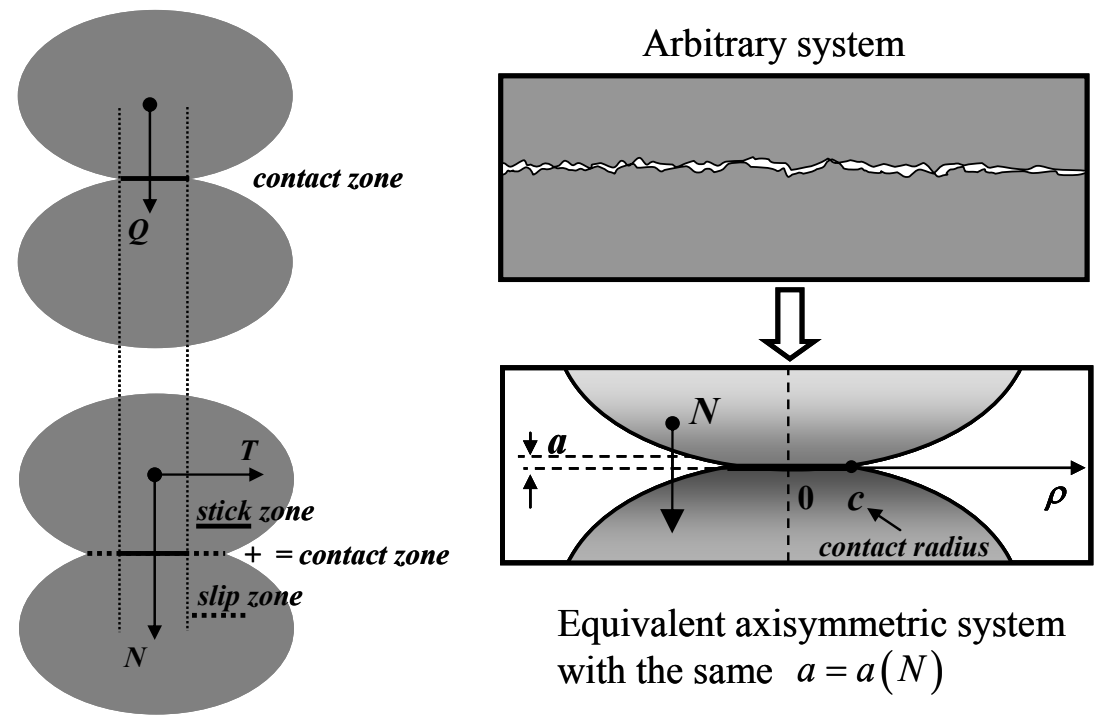

Figure 1: The reduced elastic friction principle (on the left) makes it possible to replace an arbitrary geometry with an equivalent axisymmetric profile with the same normal reaction.

$$
\left\{\begin{array}{l}
T=\mu(N-Q) \\
b=\theta \mu\left(a(N)-\left.a(N)\right|_{N=Q}\right),
\end{array}\right.
$$

where the dependency of the normal displacement on the normal force $a=a(N)$ is considered as known, and $\theta$ is a material constant that depends only on Poisson's ratio $v, \theta=(2-v) /(2(1-v))$. 
An important feature of eqn (1) is that it does not contain any geometryrelated characteristics. Thus a simple consequence of the REFP is a statement that, for two contact systems with the same normal response, the tangential responses are also identical. Consequently, a contact between surfaces of arbitrary topography can be replaced by an equivalent axisymmetric system.

\section{Method of memory diagrams}

The MMD (see [14] for detailed proofs) explained below represents an extension of the solution eq. (1) for arbitrary loading history i.e. when forces $N$ and $T$ are not constant but change in $3 \mathrm{D}$ so that loading is actually described by vector $\left(N, T_{x}, T_{y}\right)$. The principal MMD statement asserts that there exists a function $D(\vec{\eta})$ called memory function or memory diagram such as two relationships,

$$
\vec{T}=\mu \int_{0}^{N} \vec{D}(\eta) d \eta
$$

and

$$
\vec{b}=\left.\theta \mu \int_{0}^{N} \vec{D}(\eta) \frac{d a}{d N}\right|_{N=\eta} d \eta
$$

hold simultaneously. This property (called here rule I) corresponds to the force balance equation; the contact friction force equilibrates the external tangential force. In addition, two other statements are valid. The second one (rule II) claims that $|\vec{D}(\eta)| \leq 1$ that eventually follows from the Coulomb friction law.

The rule III reflects the fact that slip (if occurs) propagates from the contact boundary (weakly compressed zone for convex contact shapes) towards the contact centre (strongly compressed zone). As far as memory diagrams are concerned, this rule suggests that a possible adjustment of the memory function $\vec{D}(\eta)$ in the purpose of complying with the rule I is made by setting $|\vec{D}(\eta)|=1$ on a final segment $Q<\eta<N$ of the memory diagram. This final segment corresponds to the presence of slip that propagates inward until, by setting of $Q$, the rule I is satisfied.

Eqns (2) and (3) are integral equations whose approximate solution can be obtained for small but final force or displacement increments. The choice of arguments depends on a physical situation, e.g. for a force-driven system $\Delta N$ and $\Delta \vec{T}$ are known while $\Delta a$ and $\Delta \vec{b}$ are to be calculated. Below we discuss an implementation of the method for an arbitrary 2D loading history for a forcedriven system. In that case, eqn (2) is used for updating the memory diagram while the former produces the unknown displacement via eqn (3). 


\subsection{MMD: 2D implementation}

The MMD algorithm developed for final force increments for 2D loading i.e. when normal and tangential forces always lay in one plane is shown in fig. 2. The algorithm includes two binary choices. Firstly, if $\Delta N$ is positive (set (a)), the memory diagram extends by the respective interval $N<\eta<N+\Delta N$ on which the memory function has to be defined keeping the equality eqn. (2) (in $2 \mathrm{D}$ case vector signs in eqns. (2) and (3) have to be omitted). Further, if $|\Delta T|<\mu N$, eqn. (2) can be satisfied by updating the memory function only on the new interval as indicated in set (b), so that the area under the newly defined memory function equals $\Delta T / \mu$. In contrast, if the latter inequality does not hold, even allocating the maximum possible area on the new interval is not enough to compensate for $\Delta T$ (set (c)). Indeed, in set (c), those maximum area is $\Delta T_{1} / \mu=\Delta N$

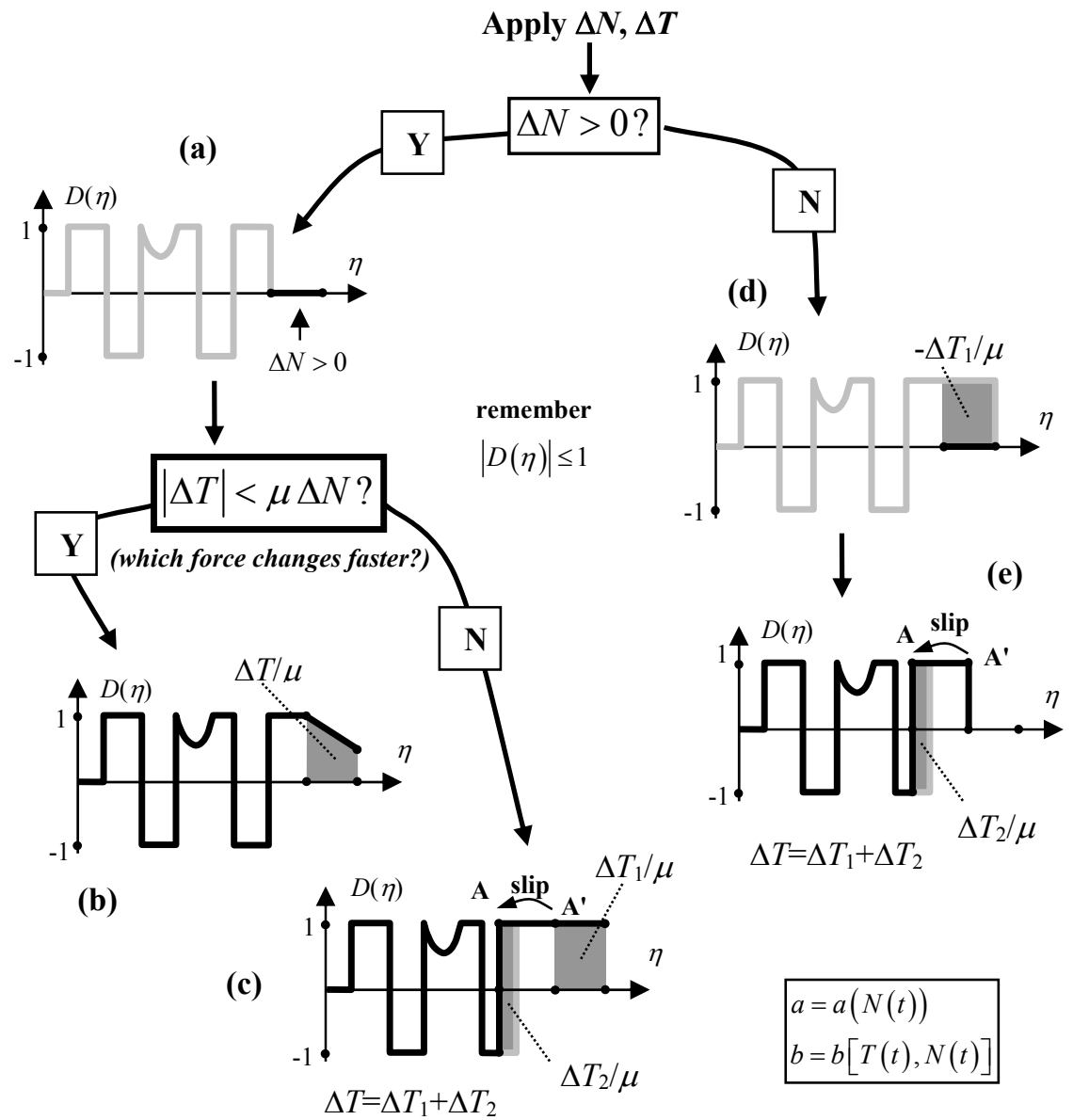

Figure 2: $\quad$ MMD algorithm in 2D. 
because of the rule II, $|D(\eta)| \leq 1$, and no more tangential force can be "absorbed". In order to take into account the remaining part $\Delta T_{2}=\Delta T-\Delta T_{1}$, we have nothing but to use rule III that explains how to update the memory function by assuming slip i.e. segment on which $|D(\eta)|=1$. In accordance to that rule, point $\mathrm{A}^{\prime}$ moves to the left and sets $|D(\eta)|=1$ at its right until the remaining force increment $\Delta T_{2}$ is equilibrated.

In the case when the inequality $\Delta N>0$ is not fulfilled, the memory diagram shrinks by the value of $|\Delta N|$ thus releasing some tangential force $\Delta T_{1}$ previously "saved" in the contact system (set (d)). The remaining value $\Delta T_{2}=\Delta T-\Delta T_{1}$ is equilibrated by assuming the slip propagation as before (set (e)).

The application of the algorithm shown in fig. 2 allows us to update the memory diagram for known force increments $\Delta N$ and $\Delta T$ in such a way that the force balance eqn (2) is kept. Then the updated memory diagram is used for calculating the tangential displacement according to eqn (3).

The above reasoning shows that a memory diagram can contain both straight segments with $|D(\eta)|=1$ corresponding to slip and curvilinear segments obtained in a situation (b) (in fig. 2 the increment values are highly exaggerated). This suggests an economic way of representing a memory diagram for numerical implementation (see fig. 3). We introduce two kinds of nodes, black and grey, and define the memory function between them. Black point $\left(\eta_{i}, D_{i}\right)$ indicates that on the interval $\eta_{i-1}<\eta<\eta_{i}$ function $D$ equals $D_{i}$; grey point $\left(\eta_{i}, D_{i}\right)$ indicates that on the interval $\eta_{i-1}<\eta<\eta_{i}$ function $D$ has to be interpolated between points $\left(\eta_{i-}\right.$ $\left.{ }_{1}, D_{i-1}\right)$ and $\left(\eta_{i}, D_{i}\right)$ using some interpolation method (linear or of higher order). Saving grey memory points is a memory-consuming process since the distances between the points correspond to force increments $\Delta N$ and are small. As for the black segments in fig. 3, only the limiting points are saved that makes the scheme much more efficient in comparison to, say, a simple equidistant grid of fixed points.

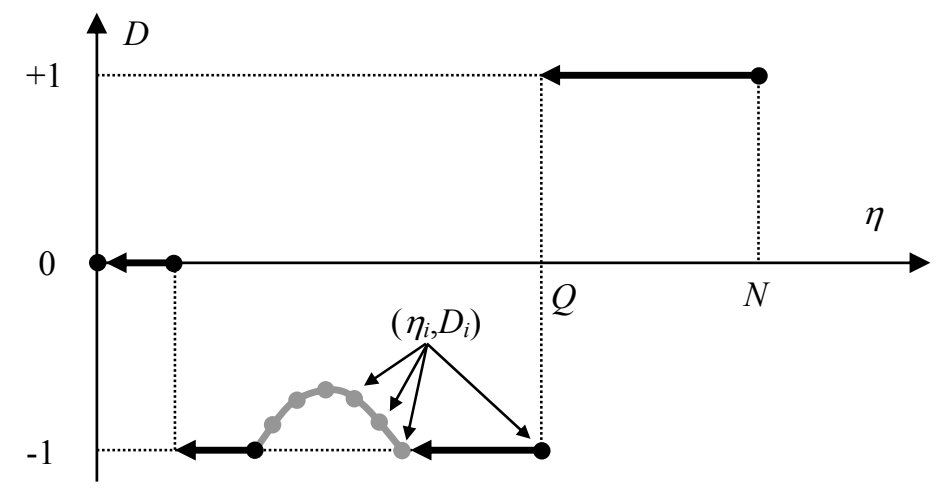

Figure 3: Possible discrete representation of the memory diagram. 
Compensating for $\Delta T$ at each step of the algorithm is possible via explicit computation. Indeed, adjustment of the length of the memory diagram according to a current increment $\Delta N$ and calculation of the related $\Delta T_{1}$ is an explicit operation since at each interval $\eta_{i-1}<\eta<\eta_{i}$, function $D(\eta)$ is given by an analytical expression. Similarly, in order to account for slip and to calculate $Q$ (the boundary between stick and slip zones, see fig. 3) we successively consider segments $\eta_{i-1}<\eta<\eta_{i}$ with reducing $i$ until the segment containing $Q$ is found. Then the actual $Q$ is analytically evaluated.

\subsection{MMD: 3D implementation}

A 3D implementation of the MMD is similar except the memory diagram consists of two functions $D_{x}(\eta)$ and $D_{y}(\eta)$ (fig. 4). The algorithm first adjusts the length of the memory diagram and calculates the related $\Delta \vec{T}_{1}$. If now the total increment $\Delta \vec{T}$ is not fully equilibrated, slips starts propagating from the contact boundary $\eta=N$ towards the centre $\eta=0$. The remaining unbalanced force $\Delta \vec{T}_{2}=\Delta \vec{T}-\Delta \vec{T}_{1}$ is known and we have to compensate for it by finding a proper $Q$. To do so, first an interval (black or grey, see the interpolation rule above) that contains $Q$ has to be found. Despite the number of equations now equals two but not one as in the $2 \mathrm{D}$ case, the interval is still possible to locate explicitly. In that purpose, we introduce the angle $\gamma$ of the slip direction and write the force balance equation in the form

$$
\left\{\begin{array}{l}
\Delta T_{x 2}=(N-Q) \cos \gamma-\int_{Q}^{N} D_{x}^{\text {old }}(\eta) d \eta \\
\Delta T_{y 2}=(N-Q) \sin \gamma-\int_{Q}^{N} D_{y}^{\text {old }}(\eta) d \eta
\end{array}\right.
$$

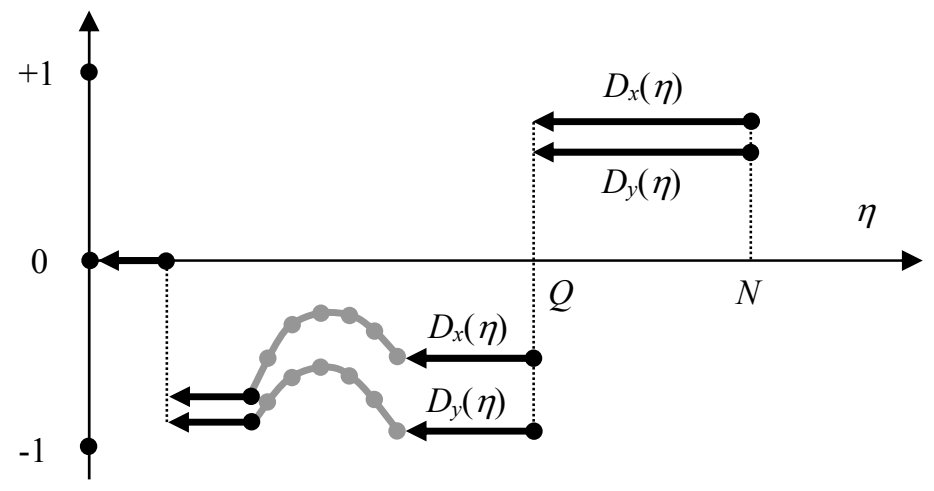

Figure 4: Vector memory function components $D_{x}(\eta)$ and $D_{y}(\eta)$ in the 3D loading case. 
where $D_{x, y}^{\text {old }}(\eta)$ is the previous (i.e. not yet updated at this step) memory diagram. By excluding $\gamma$ we obtain a single equation and then consider function

$$
F=\left(\Delta T_{x 2}+\int_{Q}^{N} D_{x}^{\text {old }}(\eta) d \eta\right)^{2}+\left(\Delta T_{x 2}+\int_{Q}^{N} D_{y}^{\text {old }}(\eta) d \eta\right)^{2}-(N-Q)^{2}
$$

that has different signs at the ends of the interval containing $Q$. The final computation of actual $Q$ on that segment is done analytically since the memory function $\vec{D}(\eta)$ is explicitly given.

\section{Results and examples}

Below we show a number of curves $b(T)$ for some input dependencies $N(t)$ and $T(t)$ in the 2D loading case. Fig. 5 illustrates a particular case when two arguments, $N$ and $T$, are linked via a functional relation, so that actually there is only one independent argument in the system. Curve $b(T)$ in fig. 5 is typical for one-parametric hysteresis [10-12], e.g. it exhibits closed loops for periodic $T(t)$; partial increase in the argument $T$ on the globally decreasing branch results in appearance of an inner loop, etc. Note that the inner loop has the property of the end-point memory which means that the curve exits the loop with the same tangent as just before entering it.

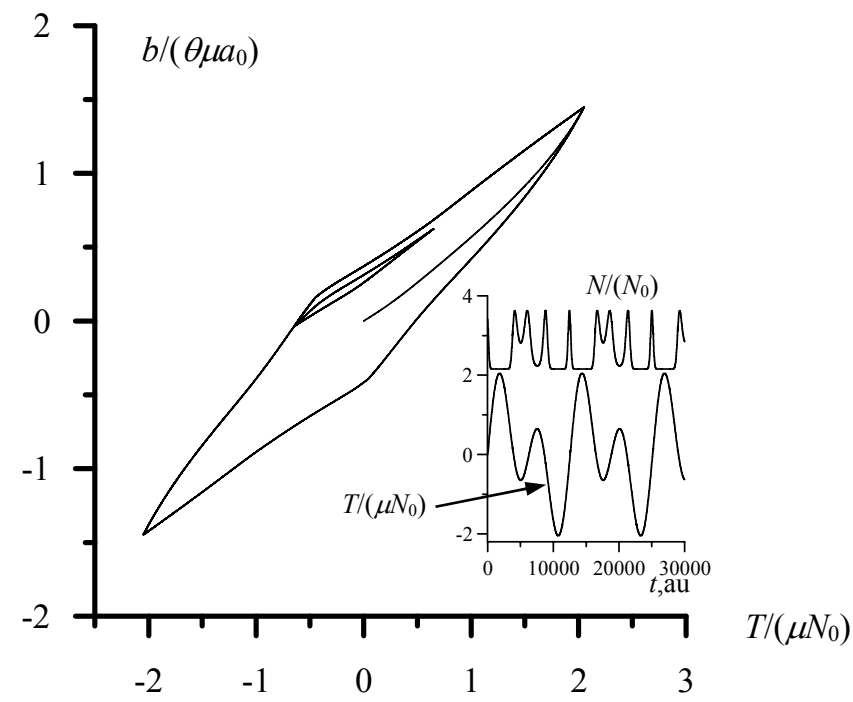

Figure 5: Tangential force-displacement curves for particular loading histories shown in the inset, in the case when $N$ and $T$ are linked via functional dependency (one-parametric hysteresis). Henceforth $N_{0}$ denotes a characteristic value of the normal force, and $a_{0}=a\left(N_{0}\right)$. 


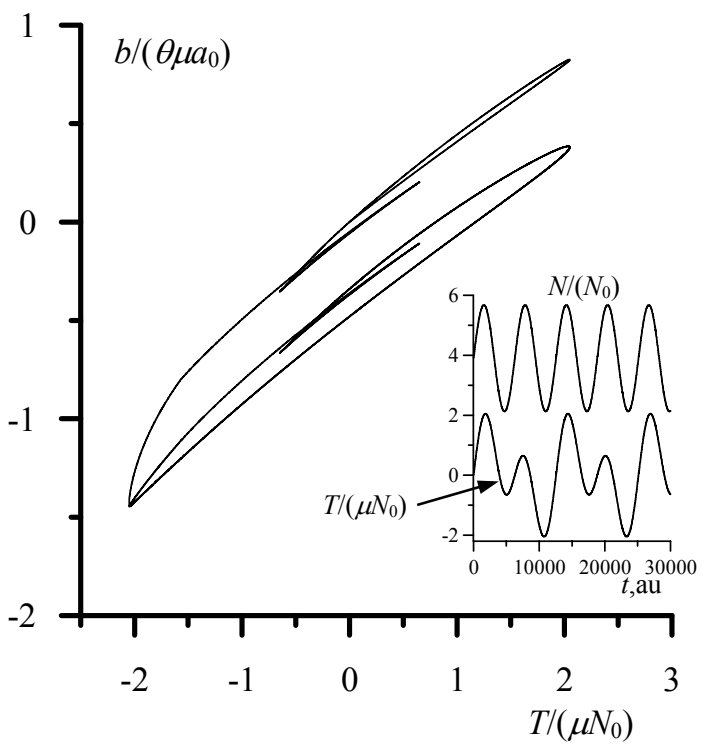

Figure 6: Tangential force-displacement curves for particular loading histories shown in the inset, in the case when $N$ and $T$ are independent (twoparametric hysteresis).

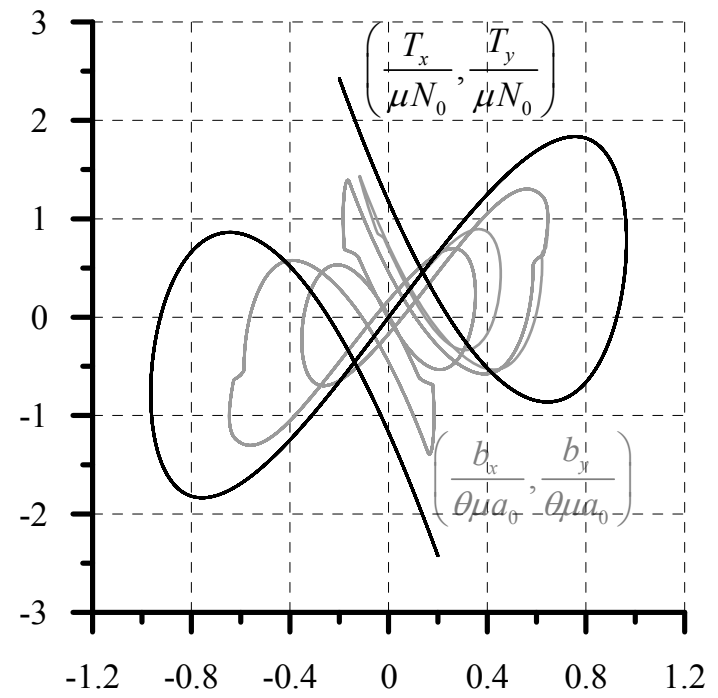

Figure 7: Input force curve $\left(T_{x}, T_{y}\right)$ (black, normalized on $\left.\mu N_{0}\right)$ and output displacement curve $\left(b_{x}, b_{y}\right)$ (gray, normalized on $\left.\theta \mu a_{0}\right)$. 
However, in a more general case when two arguments, $N$ and $T$, are independent, the hysteretic behaviour differs considerably. Since variations in $N$ are not linked with $T(t)$-protocol, even for periodic $T(t)$ the "loops" are not closed. Indeed, fig. 6 shows that the same $T(t)$-history produces a curve in which all monotonous parts are shifted, bent, etc. Of course, generating such curves via the direct analysis of traction and without use of the MMD is an extremely cumbersome task.

In the case of loading in $3 \mathrm{D}$ is more appropriate to show the solution $\left(b_{x}, b_{y}\right)$ for the excitation $\left(T_{x}, T_{y}\right)$ on the same plot (fig. 7). Here time dependences of all three force components $\left(T_{x}, T_{y}, N\right)$ are harmonic with different periods. Again, the displacement curve has a complex behaviour; its calculation by means of the MMD is fully automated.

\section{Discussion}

Below there are some remarks that concern the solution to the contact problem for an arbitrary 3D loading presented here.

a) The method discussed here assumes that the normal solution $a=a(N)$ is known. Such solutions for some regular contact geometries can be found in books $[15,16]$ on contact mechanics. For randomly rough surfaces, we refer the reader to recent papers [17-20] (see also [14] for a possible derivation of $a=a(N))$.

b) All geometry-related features are taken into account by the normal solution $a=a(N)$; the rest of the theory is formulated independently of contact geometry. The possibility of doing so follows from the reduced elastic friction principle.

c) This principle assumes some conditions discussed in [5-9], such as neglect of the effects of dissimilarity between the bodies, etc.

d) Other assumptions are similar to those of the Hertz-Mindlin theory. They include purely elastic deformations, absence of adhesion, absence of torque, plane contact, aligned and non-rotating normals to all contact spots, etc. In fact, the present method can be considered as an extension of the Hertz-Mindlin theory for an arbitrary 3D loading and a wide range of geometries.

e) Although the solution is computer-assisted, we still can consider it as analytical for final force (or displacement) increments. The algorithm shown in fig. 2 just selects an appropriate branch of the analytical solution in accordance to the relationships between the force or displacement increments.

f) As many other models in theoretical contact mechanics, our theory assumes the friction coefficient to be a universal constant for two materials in contact.

g) The theory can be applied as a basis for a description of materials with internal contacts (geomaterials or nondestructive testing of damaged pieces or components), as well as for designing new nonlinear metamaterials with desired properties. 


\section{Acknowledgements}

The authors gratefully acknowledge the support of the French National Research Agency ANR (ANL-MEMS 2010 BLAN 923 01) and of the European Commission (A life-cycle autonomous modular system for aircraft material state evaluation and restoring system, ALAMSA - FP7-AAT-2012-RTD-1, grant agreement number 314768).

\section{References}

[1] Landau, L.D. \& Lifshitz, E.M., Theory of Elasticity, Pergamon Press: Oxford, 1993.

[2] Cattaneo, C., Sul contatto di due corpi elastici: distribuzione locale degli sforzi. Accad. Lincei Rend., 27(6) pp. 342-348, 1938.

[3] Mindlin, R.D., Compliance of elastic bodies in contact. J. Appl. Mech., 16, pp. 259-268, 1949.

[4] Mindlin, R.D. \& Deresiewicz, H., Elastic spheres in contact under varying oblique forces. J. Appl. Mech., 20, pp. 327-344, 1953.

[5] Jäger, J., Axisymmetric bodies of equal material in contact under torsion or shift. Archive of Applied Mechanics, 65, pp. 478-487, 1995.

[6] Jäger, J., Half-planes without coupling under contact loading. Archive of Applied Mechanics, 67, pp. 247-259, 1997.

[7] Jäger, J., Properties of equal bodies in contact with friction. Int. J. Solids Struct., 40, pp. 5051-5061, 2003.

[8] Ciavarella, M., The generalized Cattaneo partial slip plane contact problem. I - Theory, II - Examples. Int. J. Solids Struct., 35, pp. 23492362, 1998.

[9] Ciavarella, M., Tangential loading of general 3D contacts. ASME J. Appl. Mech., 65, pp. 998-1003, 1998.

[10] Aleshin, V.V. \& Van Den Abeele, K., Preisach analysis of the HertzMindlin system. J. Mech. Phys. Solids, 57, pp. 657-672, 2009.

[11] Aleshin, V.V. \& Van Den Abeele, K., Hertz-Mindlin problem for arbitrary oblique 2D loading: General solution by memory diagrams. J. Mech. Phys. Solids, 60, pp. 14-36, 2012.

[12] Aleshin, V.V. \& Van Den Abeele, K., General solution to the HertzMindlin problem via Preisach formalism. Int. J. Non-Linear Mech., 49, pp. 15-30, 2013.

[13] Boltachev, G.Sh. \& Aleshin, V.V., Shift and torsion contact problems for arbitrary axisymmetric normal stress distributions. Int. J. Sol. Struct., 50, pp. 2894-2900, 2013.

[14] Aleshin, V.V. Bou Matar, O. \& Van Den Abeele, K., Method of memory diagrams for mechanical frictional contacts subject to arbitrary 2D loading. Int. J. Sol. Struct., 60-61, pp. 84-95, 2015.

[15] Johnson, K.L., Contact Mechanics, Cambridge University Press: Cambridge, 1985. 
478 High Performance and Optimum Design of Structures and Materials II

[16] Popov, V.L. \& Heß, M., Method of Dimensionality Reduction in Contact Mechanics and Friction, Springer Heidelberg; New York, Dordrecht, London, 2015.

[17] Hyun, S. \& Robbins, M.O., Elastic contact between rough surfaces: Effect of roughness at large and small wavelengths. Tribol. Int., 40, pp. 14131422, 2007.

[18] Carbone, G. \& Bottiglione, F., Asperity contact theories: do they predict linearity between contact area and load? J. Mech. Phys. Solids, 56, pp. 2555-2572, 2008.

[19] Paggi, M., Pohrt. R. \& Popov, V.L., Partial-slip frictional response of rough surfaces. Scientific Reports, 4, p. 5178, 2014.

[20] Paggi, M. \& Ciavarella, M., The coefficient of proportionality $\kappa$ between real contact area and load, with new asperity models. Wear, 268, pp. 1020-1029, 2010. 